\title{
On the First Homology of the Groups of Foliation Preserving Diffeomorphisms for Foliations with Singularities of Morse Type
}

By

\author{
Kazuhiko FUKU**
}

\begin{abstract}
Let $\mathbf{R}^{n}$ be an $n$-dimensional Euclidean space and $\mathcal{F}_{\varphi}$ be the foliation defined by levels of a Morse function $\varphi: \mathbf{R}^{n} \rightarrow \mathbf{R}$. We determine the first homology of the identity component of the foliation preserving diffeomorphism group of $\left(\mathbf{R}^{n}, \mathcal{F}_{\varphi}\right)$. Then we can apply it to the calculation of the first homology of the foliation preserving diffeomorphism groups for codimension one compact foliations with singularities of Morse type.
\end{abstract}

\section{$\S 1 . \quad$ Introduction and Statement of Results}

Let $\mathcal{F}$ be a $C^{\infty}$-foliation (with or without singularities) on a $C^{\infty}$-manifold $M$. Let $D^{\infty}(M, \mathcal{F})$ denote the group of all foliation preserving $C^{\infty}$ diffeomorphisms of $(M, \mathcal{F})$ which are isotopic to the identity through foliation preserving $C^{\infty}$-diffeomorphisms with compact support. In [2], we have studied the structure of the first homology of $D^{\infty}(M, \mathcal{F})$ for compact Hausdorff foliations $\mathcal{F}$, and we have that it describes the holonomy structures of isolated singular leaves of $\mathcal{F}$. Here the first homology group $H_{1}(G)$ of a group $G$ is defined as the quotient of $G$ by its commutator subgroup.

In this paper we treat the foliation preserving $C^{\infty}$-diffeomorphism groups for codimension one foliations with singularities.

Communicated by K. Saito. Received February 7, 2007. Revised October 22, 2007.

This research was partially supported by Grant-in-Aid for Scientific Research (No.

17540098), Japan Society for the Promotion of Science.

2000 Mathematics Subject Classification(s): 58D05; 57R30.

* Department of Mathematics, Kyoto Sangyo University, Kyoto 603-8555, Japan.

e-mail: fukui@cc.kyoto-su.ac.jp 
Let $\mathbf{R}^{n}$ be an $n$-dimensional Euclidean space and $\varphi_{r}: \mathbf{R}^{n} \rightarrow \mathbf{R}$ the Morse function of index $r$ defined by

$$
\varphi_{r}(x, y)=-\left(x_{1}\right)^{2}-\cdots-\left(x_{r}\right)^{2}+\left(y_{1}\right)^{2}+\cdots+\left(y_{n-r}\right)^{2},
$$

where $(x, y)=\left(x_{1}, \cdots, x_{r}, y_{1}, \cdots, y_{n-r}\right)$ is a coordinate of $\mathbf{R}^{n}=\mathbf{R}^{r} \times \mathbf{R}^{n-r}$. Let $\mathcal{F}_{\varphi_{r}}$ be the foliation defined by levels of $\varphi_{r}$, that is, $\mathcal{F}_{\varphi_{r}}$ has leaves of the form $L_{c}=\varphi_{r}^{-1}(c) \quad(c \in \mathbf{R})$. Note that $\mathcal{F}_{\varphi_{r}}$ has the only one singular leaf $L_{0}=\varphi_{r}^{-1}(0)$ through the origin. Any foliation preserving $C^{\infty}$-diffeomorphism $f:\left(\mathbf{R}^{n}, \mathcal{F}_{\varphi_{r}}\right) \rightarrow\left(\mathbf{R}^{n}, \mathcal{F}_{\varphi_{r}}\right)$ induces a homeomorphism $h$ of the leaf space $\mathbf{R}^{n} / \mathcal{F}_{\varphi_{r}}$. For the case $r \neq 1, n-1, \mathbf{R}^{n} / \mathcal{F}_{\varphi_{r}}$ is homeomorphic to the real line $\mathbf{R}$ or the half line $\mathbf{R}_{\geq 0}$ via $\varphi_{r}$, but for the case $r=1, n-1, \mathbf{R}^{n} / \mathcal{F}_{\varphi_{r}}$ is homeomorphic to a space obtained from a disjoint union of two or three real lines by identifying their closed half lines suitably. Note that each real line is diffeomorphic to $\mathbf{R}$ via $\varphi_{r}$. Then $h$ restricted to each real line is a $C^{\infty}$-diffeomorphism of itself. We have the following equation

$$
\varphi_{r}(f(x, y))=h\left(\varphi_{r}(x, y)\right)
$$

on the domains of $\mathbf{R}^{n}$ which are saturated sets of a transverse curve. Remark that $h$ depends on only $f$ and coincides with the identity if and only if $f$ is a leaf preserving $C^{\infty}$-diffeomorphism. For the case $r=0, n$, any foliation preserving $C^{\infty}$-diffeomorphism $f$ induces a $\mathbf{Z}_{2}$-equivariant $C^{\infty}$-diffeomorphism $h$ of $\mathbf{R}$. For the case $r \neq 0, n$, note that if $L_{0}$ divides $\mathbf{R}^{n}$ into only two parts, the domain is the whole $\mathbf{R}^{n}$, and at most three kinds of $C^{\infty}$-diffeomorphisms of the real line are defined generally.

We call that $f$ has a compact support if $h$ has a compact support and $f$ satisfies a certain condition on the leaf direction (see $\S 2)$. By $D_{c}^{\infty}\left(\mathbf{R}^{n}, \mathcal{F}_{\varphi_{r}}\right)$ we denote the group of all foliation preserving $C^{\infty}$-diffeomorphisms of $\left(\mathbf{R}^{n}\right.$, $\mathcal{F}_{\varphi_{r}}$ ) which are isotopic to the identity through foliation preserving $C^{\infty}$ diffeomorphisms with compact support.

The purpose of this paper is to determine the first homology of $D_{c}^{\infty}\left(\mathbf{R}^{n}\right.$, $\left.\mathcal{F}_{\varphi_{r}}\right)$ and apply this result to codimension one compact foliations with singularities of Morse type.

Then we have the following.

Theorem 1.1. $H_{1}\left(D_{c}^{\infty}\left(\mathbf{R}^{n}, \mathcal{F}_{\varphi_{r}}\right)\right) \cong\left\{\begin{array}{l}\mathbf{R} \times S^{1} \text { if } n=2 \text { and } r=0, n \\ \mathbf{R} \quad \text { otherwise. }\end{array}\right.$

Remark 1.2 (Fukui [5]). Let $D_{c}^{\infty}\left(\mathbf{R}^{n}, 0\right)$ be the group of all $C^{\infty}$ diffeomorphisms of $\mathbf{R}^{n}$ preserving the origin, which are isotopic to the iden- 
tity through $C^{\infty}$-diffeomorphisms preserving the origin with compact support. Then we have $H_{1}\left(D_{c}^{\infty}\left(\mathbf{R}^{n}, 0\right)\right) \cong \mathbf{R}$.

Remark 1.3 (Abe-Fukui [1], Abe-Fukui-Miura [4]). Let $\mathcal{H}_{L I P}\left(\mathbf{R}^{n}, 0\right)$ be the identity component of the group of all Lipschitz homeomorphisms of $\mathbf{R}^{n}$ preserving the origin with compact support under the compact open Lipschitz topology. Then we have that $\mathcal{H}_{L I P}\left(\mathbf{R}^{n}, 0\right)$ is perfect. On the other hand, let $L_{c}\left(\mathbf{R}^{n}, 0\right)$ be the identity component of the group of all Lipschitz homeomorphisms of $\mathbf{R}^{n}$ preserving the origin with compact support under the compact open topology. Then we have that $H_{1}\left(L_{c}\left(\mathbf{R}^{2}, 0\right)\right)$ has continuous moduli.

Let $M$ be an $n$-dimensional compact manifold without boundary and $\mathcal{F}$ a codimension one foliation on $M$. A point $p \in M$ is called a singularity of Morse type if there is a coordinate neighborhood $(U,(x, y))(x=$ $\left.\left(x_{1}, \cdots, x_{r}\right), y=\left(y_{1}, \cdots, y_{n-r}\right)\right)$ around $p$ where it is defined a Morse function $\varphi_{r}: U \rightarrow \mathbf{R}, \varphi_{r}(x, y)=-\left(x_{1}\right)^{2}-\cdots-\left(x_{r}\right)^{2}+\left(y_{1}\right)^{2}+\cdots+\left(y_{n-r}\right)^{2}$ such that $\left.\mathcal{F}\right|_{U}$ is given by levels of $\varphi_{r}$. For $r \neq 0, n$, we have a conical leaf given by $\varphi_{r}(x, y)=0$ in $U$. Such a singular leaf is called a separatrix leaf $L_{p}$ through $p$. For $r=0, n$, a singular leaf is a singleton. Let denote by $\mathcal{S}(\mathcal{F})$ the set of such singular leaves of $\mathcal{F}$.

We suppose the following assumption (A):

(1) Any $L \in \mathcal{S}(\mathcal{F})$ has only one singularity and

(2) For each $L \in \mathcal{S}(\mathcal{F})$, there is a compact saturated neighborhood $V_{L}$ of $L$ in $M$ such that

(i) $\mathcal{S}(\mathcal{F}) \cap V_{L}=L$,

(ii) for distinct $L, L^{\prime} \in \mathcal{S}(\mathcal{F}), V_{L}$ and $V_{L^{\prime}}$ are disjoint, and

(iii) $\left.\mathcal{F}\right|_{V_{L}}$ is given by levels of $\tilde{\varphi}$, where $\tilde{\varphi}: V_{L} \rightarrow \mathbf{R}$ is a Morse function with $\left.\tilde{\varphi}\right|_{V_{L} \cap U}=\left.\varphi_{r}\right|_{V_{L} \cap U}$ for some $r(0 \leq r \leq n)$.

Such examples are given by Morse functions from $M$ to $\mathbf{R}$ or $S^{1}$ in which no two critical points lie at the same level.

By $D^{\infty}\left(M^{n}, \mathcal{F}\right)$ we denote the group of all foliation preserving $C^{\infty}$ diffeomorphisms of $\left(M^{n}, \mathcal{F}\right)$, which are isotopic to the identity through foliation preserving $C^{\infty}$-diffeomorphisms. Then we have

Theorem 1.4. Let $\mathcal{F}$ be a codimension one $C^{\infty}$ foliation with singularities of Morse type satisfying (A) on a compact manifold $M$. We suppose that all leaves of $\mathcal{F}$ are compact and have no holonomy. Then we have

$$
H_{1}\left(D^{\infty}\left(M^{n}, \mathcal{F}\right)\right) \cong \begin{cases}\mathbf{R}^{k} \times\left(S^{1}\right)^{\ell} & \text { if } n=2 \\ \mathbf{R}^{k} & \text { if } n \geq 3\end{cases}
$$


where $k$ is the number of singularities of $\mathcal{F}$ and $\ell$ is the number of singularities of index 0 and $n$.

The paper is organized as follows. In $\S 2$, we analyze the behavior of the differentials of foliation preserving diffeomorphisms of $\left(\mathbf{R}^{n}, \mathcal{F}_{\varphi_{r}}\right)$ at the origin. This plays a key role to prove Theorem 1.1. In $\S 3$, we prove Theorem 1.1 using the theorems of Sternberg [10], Abe-Fukui [2], Thurston [11], Sergeraert [8], and Theorem 2.6 (due to Tsuboi [12]). In $\S 4$ we have an application of Theorem 1.1 to codimension one compact foliations with singularities of Morse type.

\section{§2. Preliminaries}

Let $\mathcal{F}_{r}$ be the foliation of $\mathbf{R}^{n}$ defined by levels of the Morse function $\varphi_{r}$ with singularity of index $r$ and $\mathcal{G}_{r}$ be the one dimensional foliation of $\mathbf{R}^{n}$ defined by the gradient flow of $\varphi_{r}$. We write $\varphi$ in stead of $\varphi_{r}$.

We assume that $r \neq 0, n$. Any foliation preserving $C^{\infty}$-diffeomorphism $f:\left(\mathbf{R}^{n}, \mathcal{F}_{r}\right) \rightarrow\left(\mathbf{R}^{n}, \mathcal{F}_{r}\right)$ satisfies the equation

$$
\varphi(f(x, y))=h(\varphi(x, y))
$$

on a domain of $\mathbf{R}^{n}$, where $h$ is a certain $C^{\infty}$-diffeomorphism of $\mathbf{R}$ preserving the origin, which is uniquely determined by $f$. Remark that the above relation holds on the whole $\mathbf{R}^{n}$ when $r \geq 2$ and $n-r \geq 2$. The separatrix $L_{0}=\varphi^{-1}(0)$ divides $\mathbf{R}^{n}$ into two, three or four components. Indeed, the number of the components is four for $n=2$ and $r=1$, three for $n \geq 3$ and $r=1$ or $r=n-1$, and two otherwise. Take at most three regular leaves $C_{i}$ of $\mathcal{G}_{r}$ whose union meets all leaves of $\mathcal{F}_{r}$ like the figure below. Denote by $\#\left\{C_{i}\right\}$ the number of such $C_{i}$. Then we have that $\#\left\{C_{i}\right\}=3$ for $n=2$ and $r=1$, \#\{C $\left.C_{i}\right\}=2$ for $n \geq 3$ and $r=1$ or $n=r-1$, and $\#\left\{C_{i}\right\}=1$ otherwise. Any foliation preserving $C^{\infty}$-diffeomorphism $f$ induces at most three $C^{\infty}$-diffeomorphisms $h_{i}$ of $\mathbf{R}$ via $C_{i}$.

For the case $r=0, n$, each regular leaf of $\mathcal{G}_{r}$ meets all leaves of $\mathcal{F}_{r}$ except the singular leaf. In this case, we see using the holonomy map for $\mathcal{F}_{r}$ that any foliation preserving $C^{\infty}$-diffeomorphism $f$ with compact support induces a $\mathbf{Z}_{2}$-equivariant $C^{\infty}$-diffeomorphism $h$ of $\mathbf{R}$ with compact support.

Now we define the support compactness of a foliation preserving $C^{\infty}$ diffeomorphism of $\left(\mathbf{R}^{n}, \mathcal{F}_{r}\right)$ for $r \neq 0, n$. We take a saturated neighborhood $U^{\prime}$ of $L_{0}$. Then we can take a closed neighborhood $U^{\prime \prime}$ of the origin 0 satisfying that $U^{\prime} \cap \partial U^{\prime \prime}$ is not empty and is contained in a union of regular leaves of $\mathcal{G}_{r}$. Remark that $\mathcal{F}_{r}$ is a product foliation on the intersection $U=U^{\prime} \cap\left(U^{\prime \prime}\right)^{c}$ of 


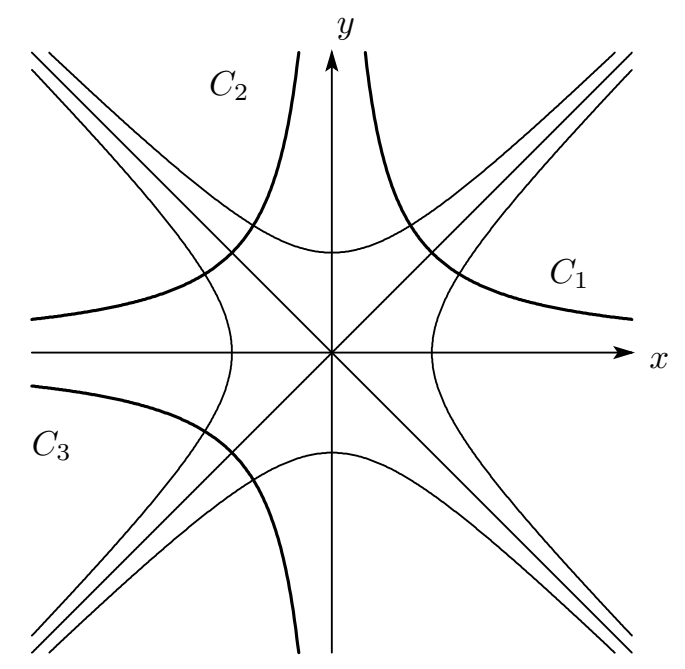

$U^{\prime}$ and the complement $\left(U^{\prime \prime}\right)^{c}$ of $U^{\prime \prime}$ by corresponding each point $p=(x, y)$ of $U$ to a pair of $\varphi(x, y) \in \mathbf{R}$ and the point of $L_{0}$ where the regular leaf of $\mathcal{G}_{r}$ passing through $p$ intersects with $L_{0}$. Then any foliation preserving $C^{\infty}$ diffeomorphism $f$ has the form $\left(f^{1}(u), f^{2}(u, v)\right)\left(\in \mathbf{R} \times\left(L_{0} \cap U\right)\right)$ on $U$, where $(u, v)$ is a foliated coordinate of $U$ such that $u=$ constant gives a leaf of $\mathcal{F}_{r}$. We fix the coordinate.

Definition 2.1. $\quad$ A foliation preserving $C^{\infty}$-diffeomorphism $f$ is said to have a compact support if $f=i d$ outside of a saturated neighborhood of $L_{0}$ and for $r \neq 0, n, f^{2}(u, v)=v$ on the intersection of the saturated neighborhood of $L_{0}$ and the complement of a neighborhood of 0 .

Let $D_{c}^{\infty}\left(\mathbf{R}^{n}, \mathcal{F}_{r}\right)$ denote the group of all foliation preserving $C^{\infty}$ diffeomorphisms of $\left(\mathbf{R}^{n}, \mathcal{F}_{r}\right)$, which are isotopic to the identity through foliation preserving $C^{\infty}$-diffeomorphisms with compact support. Let $D_{c}^{\infty}(\mathbf{R}, 0)$ denote the group of all $C^{\infty}$-diffeomorphisms of $\mathbf{R}$ preserving the origin, which are isotopic to the identity through $C^{\infty}$-diffeomorphisms preserving the origin with compact support. Let $D_{\mathbf{Z}_{2}, c}^{\infty}(\mathbf{R})$ denote the group of all $\mathbf{Z}_{2}$-equivariant $C^{\infty}$-diffeomorphisms of $\mathbf{R}$, which are isotopic to the identity through $C^{\infty}-\mathbf{Z}_{2^{-}}$ equivariant diffeomorphisms with compact support.

Set $G=D_{c}^{\infty}\left(\mathbf{R}^{n}, \mathcal{F}_{r}\right)$ and set $G_{0}=D_{\mathbf{Z}_{2}, c}^{\infty}(\mathbf{R})$, and $G_{1}=D_{c}^{\infty}(\mathbf{R}, 0), G_{2}=$ $\left\{\left(h_{1}, h_{2}\right) \in D_{c}^{\infty}(\mathbf{R}, 0) \times D_{c}^{\infty}(\mathbf{R}, 0) \mid h_{1}=h_{2}\right.$ on $\left.\mathbf{R}_{\geq 0}\right\}$, and $G_{3}=\left\{\left(h_{1}, h_{2}, h_{3}\right) \in\right.$ $D_{c}^{\infty}(\mathbf{R}, 0) \times D_{c}^{\infty}(\mathbf{R}, 0) \times D_{c}^{\infty}(\mathbf{R}, 0) \mid h_{1}=h_{2}$ on $\mathbf{R}_{\geq 0}, h_{2}=h_{3}$ on $\left.\mathbf{R}_{\leq 0}\right\}$. 
Remark that $h_{i}^{\prime}(0)=h_{j}^{\prime}(0)$ for any $i, j$, where $h_{i}^{\prime}(0)$ is the differential of $h_{i}$ at the origin 0 .

Let $f \in G$. Then, by taking suitable regular leaves $C_{i}$ of $\mathcal{G}_{r}, f$ induces a $\mathbf{Z}_{2}$-equivariant $C^{\infty}$-diffeomorphism $h \in G_{0}$, and a $C^{\infty}$-diffeomorphism $h \in G_{1}$, a pair $\left(h_{1}, h_{2}\right) \in G_{2}$ and a triple $\left(h_{1}, h_{2}, h_{3}\right) \in G_{3}$ according to $r=0, n$, and $\#\left\{C_{i}\right\}=1,2$ and 3 respectively. Then we define the map $d_{1}: G \rightarrow G_{i}$ by $d_{1}(f)=h$ when $r=0, n, d_{1}(f)=h$ when $\#\left\{C_{i}\right\}=1, d_{1}(f)=\left(h_{1}, h_{2}\right)$ when $\#\left\{C_{i}\right\}=2$, and $d_{1}(f)=\left(h_{1}, h_{2}, h_{3}\right)$ when $\#\left\{C_{i}\right\}=3$ respectively, for any $f \in G$. Then we have the following lemma.

Lemma 2.2. $\quad d_{1}$ is a surjective homomorphism.

Proof. It is clear that $d_{1}$ is a homomorphism. First we prove the surjectivity of $d_{1}$ for the case $\#\left\{C_{i}\right\}=1$. Take $h \in G_{1}$. Then there is a $C^{\infty}$-mapping $g: \mathbf{R} \rightarrow \mathbf{R}$ satisfying that $h(t)=g(t) t$ for $t \in \mathbf{R}$. Note that $g(t)>0$ for any $t \in \mathbf{R}, g(0)=h^{\prime}(0)$ and $g(t)=1$ on the complement of the support of $h$. Hence $\sqrt{g(t)}$ also becomes a $C^{\infty}$-mapping of $\mathbf{R}$ to $\mathbf{R}$. Then we define a $C^{\infty}$-mapping $\tilde{h}: \mathbf{R}^{n} \rightarrow \mathbf{R}^{n}$ by

$$
\tilde{h}(x, y)=\sqrt{g(\varphi(x, y))}(x, y) \quad \text { for }(x, y) \in \mathbf{R}^{r} \times \mathbf{R}^{n-r}=\mathbf{R}^{n} .
$$

Then we see that $\tilde{h}$ is a $C^{\infty}$-diffeomorphism and satisfies the equation

$$
\varphi \circ \tilde{h}(x, y)=h \circ \varphi(x, y)
$$

for $(x, y) \in \mathbf{R}^{r} \times \mathbf{R}^{n-r}=\mathbf{R}^{n}$, hence it is foliation preserving. By modifying $\tilde{h}$ along the leaves on the intersection of a saturated neighborhood of $L_{0}$ and the complement of a neighborhood of 0 appropriately, we may assume $\tilde{h} \in G$. Hence $d_{1}$ is surjective. When $r=0, n$, it is proved similarly as in the proof of the case $\#\left\{C_{i}\right\}=1$ by noting $g(-t)=g(t)(t \in \mathbf{R})$ for any $h \in G_{0}$.

Next we prove the surjectivity of $d_{1}$ for the case $\#\left\{C_{i}\right\}=2$. Take $\left(h_{1}, h_{2}\right) \in$ $G_{2}$. As in the proof of the case $\#\left\{C_{i}\right\}=1$, we can take $\tilde{h}_{i}$ on the union $U_{i}$ of leaves intersecting $C_{i}$ for each $h_{i}(i=1,2)$. Note that since $h_{1}=h_{2}$ on $\mathbf{R}_{\geq 0}$, $\tilde{h}_{1}=\tilde{h}_{2}$ on $U_{1} \cap U_{2}$. Therefore we can define $\tilde{h} \in G$ by $\tilde{h}=\tilde{h}_{i}$ on $U_{i}$. Then we have $d_{1}(\tilde{h})=\left(h_{1}, h_{2}\right) \in G_{2}$.

It is proved similarly for the case $\#\left\{C_{i}\right\}=3$. This completes the proof.

Next we define a map $d_{2}: G \rightarrow G L_{+}(n, \mathbf{R})$ as follows. For any $f \in G$, we take $h \in G_{i}(i=0,1),\left(h_{1}, h_{2}\right) \in G_{2}$ or $\left(h_{1}, h_{2}, h_{3}\right) \in G_{3}$ as above and consider lifts $\tilde{h}$ or $\tilde{h}_{i}$ of $h$ or $\left(h_{1}, h_{2}\right),\left(h_{1}, h_{2}, h_{3}\right)$ as in Lemma 2.2, and take the differential of $f \circ \tilde{h}^{-1}$ or $f \circ \tilde{h}_{i}^{-1}$ at the origin 0 . Then we define the map 
$d_{2}: G \rightarrow G L_{+}(n, \mathbf{R})$ by $d_{2}(f)=d\left(f \circ \tilde{h}^{-1}\right)(0)$ or $d\left(f \circ \tilde{h}_{i}^{-1}\right)(0)$. Remark that the differential of $\tilde{h}_{i}$ at the origin 0 is equal to $\sqrt{h_{i}^{\prime}(0)} I_{n}$ and $h_{i}^{\prime}(0)=h_{j}^{\prime}(0)$, where $I_{n}$ denotes the unit matrix.

Lemma 2.3. $\quad d_{2}$ is a homomorphism.

Proof. For $f_{1}, f_{2} \in G$, we take $d_{1}\left(f_{1}\right), d_{1}\left(f_{2}\right)$ and their lifts $\tilde{h}_{1}, \tilde{h}_{2}$ as above. Since $d \tilde{h}_{1}(0)=\sqrt{d_{1}\left(f_{1}\right)^{\prime}(0)} I_{n}$ and $d \tilde{h}_{2}(0)=\sqrt{d_{1}\left(f_{2}\right)^{\prime}(0)} I_{n}$, we have

$$
\begin{aligned}
d\left(f_{2} \circ f_{1}\right) & =d_{2}\left(f_{2} \circ f_{1} \circ\left(\tilde{h}_{2} \circ \tilde{h}_{1}\right)^{-1}\right) \\
& =d f_{2}(0) \cdot d f_{1}(0) \cdot d \tilde{h}_{1}^{-1}(0) \cdot d \tilde{h}_{2}^{-1}(0) \\
& =d f_{2}(0) \cdot d f_{1}(0) \cdot \frac{1}{\sqrt{d_{1}\left(f_{1}\right)^{\prime}(0)}} I_{n} \cdot \frac{1}{\sqrt{d_{1}\left(f_{2}\right)^{\prime}(0)}} I_{n} \\
& =d f_{2}(0) \cdot \frac{1}{\sqrt{d_{1}\left(f_{2}\right)^{\prime}(0)}} I_{n} \cdot d f_{1}(0) \cdot \frac{1}{\sqrt{d_{1}\left(f_{1}\right)^{\prime}(0)}} I_{n} \\
& =d_{2}\left(f_{2} \circ \tilde{h}_{2}^{-1}\right) \cdot d_{2}\left(f_{1} \circ \tilde{h}_{1}^{-1}\right) \\
& =d\left(f_{2}\right) \circ d\left(f_{1}\right) .
\end{aligned}
$$

Thus $d_{2}$ is a homomorphism. This completes the proof.

Let $S O(r, n-r)=\left\{A \in M(n, \mathbf{R}) ;{ }^{t} A I_{r, n-r} A=I_{r, n-r}, \operatorname{det} A=1\right\}$ and $S O(r, n-r)_{0}$ be its connected component containing the unit matrix, where $I_{r, n-r}=\left(\begin{array}{cc}-I_{r} & 0 \\ 0 & I_{n-r}\end{array}\right)$.

Lemma 2.4. $\operatorname{Im} d_{2}=S O(r, n-r)_{0}$.

Proof. Take $f \in G$ and a lift $\tilde{h}$ of $d_{1}(f)$ as in Lemma 2.2. Since $d_{1}(f)=$ $d_{1}(\tilde{h}), k=f \circ \tilde{h}^{-1}$ is leaf preserving. Thus we have the equation

$$
\varphi(k(x, y))=\varphi(x, y)
$$

for $(x, y) \in \mathbf{R}^{r} \times \mathbf{R}^{n-r}$. Then we have that $d_{2}(f) \in S O(r, n-r)_{0}$ by differentiating (1) with respect to $x$ and $y$ and taking the limit as $x$ and $y$ tend to 0 .

Conversely, any $A \in S O(r, n-r)_{0}$ acts on $\mathbf{R}^{n}$ mapping each leaf of $\mathcal{F}_{r}$ to itself. Then by deforming $A$ outside of a saturated neighborhood of $L_{0}$ to the identity and adjusting along the leaves on the intersection of a neighborhood of 
$L_{0}$ and the complement of a neighborhood of 0 appropriately, we have $f_{A} \in G$ satisfying $f_{A}=A$ on a neighborhood of 0 . This completes the proof.

Let $d: G \rightarrow G_{i} \times S O(r, n-r)_{0}$ be the map defined by $d(f)=\left(d_{1}(f), d_{2}(f)\right)$ for any $f \in G$. Then we have the following from Lemmas 2.2, 2.3 and 2.4.

Corollary 2.5. $\quad d$ is a surjective homomorphism.

Proof. Take $(h, A) \in G_{i} \times S O(r, n-r)_{0}$. From Lemma 2.2, we have $\tilde{h} \in G$ with $d_{1}(\tilde{h})=h$. For $A \in S O(r, n-r)_{0}$, we construct $f_{A} \in G$ as in the proof of Lemma 2.4. Then since $d_{2}(\tilde{h})=I_{n}$ and $f_{A}$ is leaf preserving, the composition $f_{A} \circ \tilde{h}$ satisfies $d\left(f_{A} \circ \tilde{h}\right)=(h, A)$. This completes the proof.

Let $\mathcal{F}_{0}$ be the product foliation of $\mathbf{R}^{m}$ with leaves of form $\left\{\{x\} \times \mathbf{R}^{m-q}\right\}$, where $(x, y)$ is a coordinate of $\mathbf{R}^{m}=\mathbf{R}^{q} \times \mathbf{R}^{m-q}$. By $D_{L, c}^{\infty}\left(\mathbf{R}^{m}, \mathcal{F}_{0}\right)$ we denote the group of leaf preserving $C^{\infty}$-diffeomorphisms of $\left(\mathbf{R}^{m}, \mathcal{F}_{0}\right)$ which are isotopic to the identity through leaf preserving $C^{\infty}$-diffeomorphisms with compact support.

T.Tsuboi [12] (and T.Rybicki [7]) proved the following by looking at the proofs in Herman [6] and Thurston [11].

Theorem 2.6 (Theorem 1.1 of $[12]) . \quad D_{L, c}^{\infty}\left(\mathbf{R}^{m}, \mathcal{F}_{0}\right)$ is perfect.

\section{$\S 3 . \quad$ Proof of Theorem 1.1}

From Corollary 2.5, for each $i(i=0,1,2,3)$ there is a short exact sequence

$$
1 \longrightarrow \operatorname{ker} d \longrightarrow G \stackrel{d}{\longrightarrow} G_{i} \times S O(r, n-r)_{0} \longrightarrow 1 .
$$

Thus we have the following exact sequence of homology groups:

$$
\text { ker } d /[\text { ker } d, G] \longrightarrow H_{1}(G) \stackrel{d_{*}}{\longrightarrow} H_{1}\left(G_{i} \times S O(r, n-r)_{0}\right) \longrightarrow 1 \text {. }
$$

First we shall prove $\operatorname{ker} d /[\operatorname{ker} d, G]=0$.

Proposition 3.1. $\quad \operatorname{ker} d=[\operatorname{ker} d, G]$.

Proof. Take any $f \in \operatorname{ker} d$. Note that $f$ is leaf preserving. Take an expansion $L_{c}$ defined by $L_{c}(x, y)=(c x, c y)$ for $(x, y) \in \mathbf{R}^{n}$, where $c>1$. Then $L_{c}$ is foliation preserving and we may assume that it is contained in $G$ by modifying $L_{c}$ outside of a neighborhood of 0 suitably. We consider the 
composition $f \circ L_{c}$. Then we have $d\left(f \circ L_{c}\right)(0)=c I_{n}$. From Theorem 1 of Sternberg [10], there exists $R \in D^{\infty}\left(\mathbf{R}^{n}, 0\right)$ satisfying that $d R(0)=I_{n}$ and

$$
R^{-1} \circ f \circ L_{c} \circ R=L_{c}
$$

on a neighborhood of 0 , say $V$. We prove that $R$ is leaf preserving on $V$. From (2), we have $\varphi \circ R(x, y)=\varphi \circ f \circ L_{c} \circ R \circ L_{c}^{-1}(x, y)$ for $(x, y) \in V$. Since $f$ is leaf preserving and $\varphi$ is a quadratic form, we have

$$
\varphi(R(x, y))=c^{2}\left(\varphi\left(R\left(\frac{1}{c} x, \frac{1}{c} y\right)\right)\right)
$$

for $(x, y) \in V$. From (3) and $d R(0)=I_{n}, R$ has the following form

$$
R(x, y)=(x, y) A(x, y),
$$

where $A: V \rightarrow G L(n, \mathbf{R})$ is a $C^{\infty}$-mapping which is 1 -tangent to the constant mapping $e_{n}\left(e_{n}(x, y)=I_{n}\right)$ at 0 . Furthermore we have

$$
\varphi((x, y) A(x, y))=\varphi\left((x, y) A\left(\frac{1}{c} x, \frac{1}{c} y\right)\right)
$$

from (3), thus

$$
\varphi((x, y) A(x, y))=\varphi\left((x, y) A\left(\frac{1}{c^{n}} x, \frac{1}{c^{n}} y\right)\right)
$$

for any positive integer $n$. By letting $n$ tend to $\infty$, we have

$$
\varphi((x, y) A(x, y))=\varphi(x, y) .
$$

Hence we have $\varphi \circ R(x, y)=\varphi(x, y)$ for $(x, y) \in V$, so $R$ is leaf preserving on $V$. Furthermore we may assume $R \in G$ by modifying $R$ outside of $V$ suitably. Therefore we have $f=\left[R, L_{c}\right]$ on $V$, where $R \in \operatorname{ker} d$ and $L_{c} \in G$.

Since $\mathcal{F}_{r}$ is a product foliation on the intersection of the complement of $V$ and a neighborhood of $L_{0}$ and $f$ has a compact support, $f \circ\left[R, L_{c}\right]^{-1}$ is written as a product of commutators of leaf preserving diffeomorphisms from Theorem 2.6. This completes the proof.

Proof of Theorem 1.1 continued. From Proposition 3.1, we have

$$
H_{1}(G) \cong H_{1}\left(S O(r, n-r)_{0} \times G_{i}\right) \cong H_{1}\left(S O(r, n-r)_{0}\right) \times H_{1}\left(G_{i}\right) .
$$

We calculate $H_{1}\left(G_{i}\right)(i=0,1,2,3)$. We have $H_{1}\left(G_{i}\right) \cong \mathbf{R}$ for $i=0,1$ from Abe-Fukui [2] and Fukui [5]. For $i=2$, we define the map $d_{3}: G_{2} \rightarrow \mathbf{R}_{>0}$ 
by taking the differential of $h_{1}$ or $h_{2}$ at 0 for any $\left(h_{1}, h_{2}\right) \in G_{2}$. Note that $h_{1}^{\prime}(0)=h_{2}^{\prime}(0)$ because that $h_{1}=h_{2}$ on $\mathbf{R}_{\geq 0}$. Then for any $\left(h_{1}, h_{2}\right) \in \operatorname{ker} d_{3}$, $h_{1}$ can be represented by a commutator of a diffeomorphism $\phi$ of the form $t \rightarrow c t(0<c<1)$ locally and an element in $G_{1}$ which is 1 -tangent to the identity at 0 , say $\psi$, on a neighborhood of 0 by the standard argument (cf. Fukui [5]). Then we may assume from the theorem of Thurston [11] that $h_{2} \circ[\phi, \psi]^{-1}$ is equal to the identity on $\mathbf{R}_{\geq 0}$, and is $C^{\infty}$-tangent to the identity at 0 . From Sergeraert $[8], h_{2} \circ[\phi, \psi]^{-1}$ can be represented by a product of commutators of diffeomorphisms which are equal to the identity on $\mathbf{R}_{\geq 0}$ and are $C^{\infty}$-tangent to the identity at 0 . Thus $\left(h_{1}, h_{2}\right)$ can be represented by a product of commutators of elements in $G_{2}$. Hence we have $H_{1}\left(G_{2}\right) \cong \mathbf{R}$. It is also proved for $i=3$ similarly.

Since $S O(r, n-r)_{0}$ is simple or semi-simple except for $n=2$ and $r=0$ or 2 , we complete the proof.

\section{§4. Proof of Theorem 1.4}

Let $p_{1}, \cdots, p_{k}$ be all singularities of $\mathcal{F}$. For each $p_{i}$, there are a compact saturated neighborhood $V_{i}$ and a Morse function $\varphi_{i}: V_{i} \rightarrow \mathbf{R}$ such that

(i) $\mathcal{S}(\mathcal{F}) \cap V_{i}=L_{p_{i}}$, where $L_{p_{i}}$ is the leaf passing through $p_{i}$,

(ii) $V_{i} \cap V_{j}=\emptyset$ if $i \neq j$, and

(iii) $\left.\mathcal{F}\right|_{V_{i}}$ is given by levels of $\varphi_{i}$.

Let $\mathcal{G}$ be a one dimensional foliation of $M$ transverse to $\mathcal{F}$ satisfying that $\left.\mathcal{G}\right|_{V_{i}}$ is defined by the gradient flow of $\varphi_{i}$. For each $i$, take at most three regular leaves $C_{j(i)}$ of $\mathcal{G}$ whose union meets all leaves of $\left.\mathcal{F}\right|_{V_{i}}$.

Let $G_{1}$ denote the group of germs at the origin 0 of elements of the group $D^{\infty}(\mathbf{R}, 0)$ whose elements are isotopic to the identity through $C^{\infty}$ diffeomorphisms preserving the origin. We denote by $G_{0}$ the group of germs at the origin 0 of elements of the group $D_{\mathbf{Z}_{\mathbf{2}}}^{\infty}(\mathbf{R})$ whose elements are isotopic to the identity through $C^{\infty}-\mathbf{Z}_{2}$-equivariant diffeomorphisms. Set $G_{2}=\left\{\left(h_{1}, h_{2}\right) \in G_{1} \times G_{1} \mid \hat{h}_{1}=\hat{h}_{2}\right.$ on $\mathbf{R}_{\geq 0}$, where $\hat{h}_{1}, \hat{h}_{2}$ are representatives of $\left.h_{1}, h_{2}\right\}$ and $G_{3}=\left\{\left(h_{1}, h_{2}, h_{3}\right) \in G_{1} \times G_{1} \times G_{1} \mid \hat{h}_{1}=\hat{h}_{2}\right.$ on $\mathbf{R}_{\geq 0}, \hat{h}_{2}=$ $\hat{h}_{3}$ on $\mathbf{R}_{\leq 0}$, where $\hat{h}_{1}, \hat{h}_{2}, \hat{h}_{3}$ are representatives of $h_{1}, h_{2}, h_{3}$ respectively $\}$.

Take any $f \in D^{\infty}\left(M^{n}, \mathcal{F}\right)$. By taking the map $d$ at each $p_{i}$ as in $\S 2$ via regular leaves $C_{j(i)}$, we have the surjective homomorphism

$$
\Psi: D^{\infty}\left(M^{n}, \mathcal{F}\right) \rightarrow \prod_{i=1}^{k}\left(G_{\ell(i)} \times S O\left(r_{i}, n-r_{i}\right)_{0}\right),
$$


where $\ell(i)=0,1,2,3$. Then we have a short exact sequence:

$$
\begin{aligned}
& 1 \longrightarrow \operatorname{ker} \Psi D^{\infty}\left(M^{n}, \mathcal{F}\right) \\
& \stackrel{\Psi}{\longrightarrow} \prod_{i=1}^{k}\left(G_{\ell(i)} \times S O\left(r_{i}, n-r_{i}\right)_{0}\right) \longrightarrow 1
\end{aligned}
$$

Thus we have the following exact sequence of homology groups:

$$
\begin{aligned}
& \operatorname{ker} \Psi /\left[\operatorname{ker} \Psi, D^{\infty}\left(M^{n}, \mathcal{F}\right)\right] \longrightarrow \\
& \stackrel{\Psi_{*}}{\longrightarrow} H_{1}\left(\prod_{i=1}^{k}\left(G_{\ell(i)} \times S O\left(r_{i}, n-r_{i}\right)_{0}\right)\right) \longrightarrow 1
\end{aligned}
$$

Since any $g \in \operatorname{ker} \Psi$ is leaf preserving on a saturated neighborhood $W$ of $\bigcup_{i=1}^{k} L_{p_{i}}$ and $\mathcal{F}$ restricted to each connected component of $W^{c}=M-W$ is a bundle foliation with compact fiber, any $g \in \operatorname{ker} \Psi$ can be decomposed as $g=g_{1} \circ \cdots \circ g_{k} \circ g^{\prime}$, where the support of each $g_{i}$ is contained in $V_{i} \cap W$ and the support of $g^{\prime}$ is contained in a neighborhood of $W^{c}$. Thus from Proposition 3.1, Theorem 2.6 and Corollary 5.4 of [2], we have $\operatorname{ker} \Psi /\left[\operatorname{ker} \Psi, D^{\infty}\left(M^{n}, \mathcal{F}\right)\right]=0$. Hence we have

$$
\begin{aligned}
H_{1}\left(D^{\infty}\left(M^{n}, \mathcal{F}\right)\right) & \cong H_{1}\left(\prod_{i=1}^{k}\left(G_{\ell(i)} \times S O\left(r_{i}, n-r_{i}\right)_{0}\right)\right) \\
& \cong \prod_{i=1}^{k} H_{1}\left(G_{\ell(i)} \times S O\left(r_{i}, n-r_{i}\right)_{0}\right) .
\end{aligned}
$$

As in the proof of Theorem 1.1 continued, we can show $H_{1}\left(G_{\ell(i)}\right) \cong \mathbf{R}(i=$ $0,1,2,3)$ using the results of Abe-Fukui [2], Fukui [5] and Sergeraert [8]. The proof follows from Theorem 1.1.

\section{References}

[1] K. Abe and K. Fukui, On the structure of the group of Lipschitz homeomorphisms and its subgroups. II, J. Math. Soc. Japan 55 (2003), no. 4, 947-956.

2] On the first homology of the group of equivariant diffeomorphisms and its applications, J. of Topology 1 (2008), no. 2, 461-476.

[3] $\ldots$, Commutators of $C^{\infty}$-diffeomorphisms preserving a submanifold, to appear in J. Math. Soc. Japan.

[4] K. Abe, K. Fukui and T. Miura, On the first homology of the group of equivariant Lipschitz homeomorphisms, J. Math. Soc. Japan 58 (2006), no. 1, 1-15.

[5] K. Fukui, Homologies of the group $\operatorname{Diff}^{\infty}\left(\mathbf{R}^{n}, 0\right)$ and its subgroups, J. Math. Kyoto Univ. 20 (1980), no. 3, 475-487. 
[6] M. Herman, Simplicité du groupe des difféomorphismes de classe $C^{\infty}$, isotopes á l'identité, du tore de dimension n, C. R. Acad. Sci. Paris, Sér. A-B 273 (1971), 232-234.

[7] T. Rybicki, The identity component of the leaf preserving diffeomorphism group is perfect, Monatsh. Math. 120 (1995), no. 3-4, 289-305.

[8] F. Sergeraert, Feuilletages et difféomorphismes infiniment tangents à l'identité, Invent. Math. 39 (1977), no. 3, 253-275.

[9] S. Sternberg, Local contractions and a theorem of Poincaré, Amer. J. Math. 79 (1957), 809-824

[10] , On the structure of local homeomorphisms of euclidean $n$-space, II, Amer. J. Math. 80 (1958), 623-631.

[11] W. Thurston, Foliations and groups of diffeomorphisms, Bull. Amer. Math. Soc. 80 (1974), 304-307.

[12] T. Tsuboi, On the group of foliation preserving diffeomorphisms, in Foliations 2005, ed. by P. Walczak et al. World Scientific, Shingapore, (2006), 411-430. 